\title{
Finite volume effects in the gluon propagator
}

\author{
Orlando Oliveira* and Paulo J. Silva ${ }^{\dagger}$ \\ Centro de Física Computacional, Departamento de Física, Universidade de Coimbra \\ 3004-516 Coimbra, Portugal. \\ E-mail: orlando@teor.fis.uc.pt , psilva@teor.fis.uc.pt
}

\begin{abstract}
We report on a preliminary study of the volume dependence of the gluon propagator. The propagator is computed using different lattice volumes, its extrapolation to infinite volume is investigated with particular attention to the its IR behaviour. Our data shows a mild but measurable dependence with the volume. Unfortunately, we are not able yet to clarify its behaviour close to zero momentum.
\end{abstract}

XXIIIrd International Symposium on Lattice Field Theory

25-30 July 2005

Trinity College, Dublin, Ireland

\footnotetext{
${ }^{*}$ Speaker.

${ }^{\dagger}$ Supported by FCT via grant SFRH/BD/10740/2002.
} 


\section{Introduction and motivation}

The infrared (IR) properties of the gluon propagator were studied in [1, 2] with the following large assymetric lattices $16^{3} \times 128$ and $16^{3} \times 256$. For these lattices, although the propagator for pure temporal momenta are within errors, the equivalent spatial momenta show a deviation from the temporal ones for small momenta. Clearly, this is a finite volume effect. So the question, to which we will try to give a first and preliminary answer, is how reliable are the figures reported in $[1,2]$ ? Or what is the meaning of these results? In order to try to answer these questions, we make a first study of the volume dependence of the gluon propagator, which will also allow an extrapolation to infinite volume.

\section{Lattice setup}

In order to study the volume dependence we generated ${ }^{1} \mathrm{SU}(3)$ pure gauge, Wilson action configurations for the lattices reported in table 1. The table also describes the combined overrelaxed+heat bath Monte Carlo sweeps, the number of combined sweeps for thermalization, the separation of combined sweeps used for each simulation and the total number of configurations for each lattice.

\begin{tabular}{|r|r|r|r|r|}
\hline Lattice & Update & therm. & Sep. & Conf. \\
\hline $8^{3} \times 256$ & 7 OVR+4HB & 1500 & 1000 & 80 \\
$10^{3} \times 256$ & 7 OVR+4HB & 1500 & 1000 & 80 \\
$12^{3} \times 256$ & 7 OVR+4HB & 1500 & 1000 & 80 \\
$14^{3} \times 256$ & 7 OVR+4HB & 3000 & 1000 & 47 \\
$16^{3} \times 256$ & 7 OVR+4HB & 3000 & 1500 & 155 \\
\hline
\end{tabular}

Table 1: Lattices setup used in the study of the volume dependence.

For the definition of the gluon propagator, notation and the gauge fixing method see [3].

\section{Time versus Spatial Momenta}

Our first check was to look at how the time and the different spatial momenta evolved with the lattice volume. The gluon propagator, for the smallest and the largest lattices, for different types of spatial momenta is given in figure 1. The data shows that the propagator becomes smaller when the number of zero components increases. The effect is larger for smaller momenta. For the largest lattice, the effect is not so strong but is, nevertheless, clearly visible.

The comparison between temporal and spatial momenta with only one nonzero component shows a similar behaviour. The temporal data is always below the spatial data - see [2].

The gluon dressing function for temporal momenta and spatial momenta with only one nonzero component show a strong dependence on the volume, see figure 2, specially in the intermediate momenta region $(\sim 1 \mathrm{GeV})$. Note that while the temporal data seems to increase with the volume,

\footnotetext{
${ }^{1}$ All configurations were generated with MILC code $h t t p: / /$ physics. indiana.edu/ ${ }^{\mathrm{sg}} / \mathrm{milc} . \mathrm{htm} \quad 1$.
} 

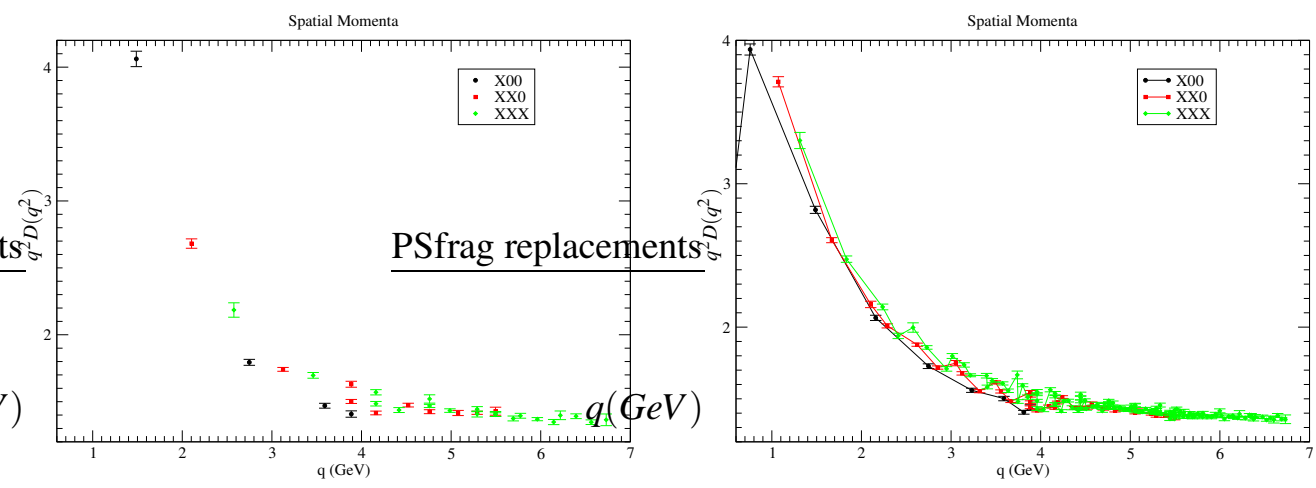

Figure 1: Dressing function for different spatial momenta, for the smaller (left) and largest (right) lattices.

the spatial data decreases with the volume. Certainly, this is the case for almost all the momenta region but not for the lowest momenta - see figure 4. Indeed, a zoom to the IR region shows the opposite behaviour, i.e. for small momenta the propagator decreases with the volume. Therefore, if in the IR the gluon dressing function is given by $\left(q^{2}\right)^{2 \kappa}$, a decreasing of the dressing function means that $\kappa$ should increase with the volume. Then, the values measured with any finite lattice can be seen, at least, as a lower bound in $\kappa$.

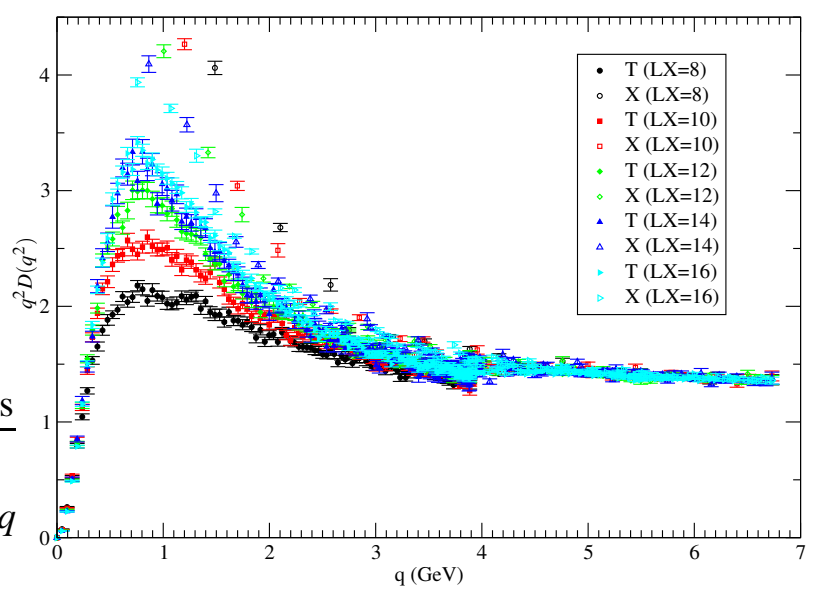

Figure 2: Gluon dressing functions

\section{IR region}

In [2] it was seen that the IR region is well described either by a pole or a cut behaviour ${ }^{2}$. We fitted both expressions to the different lattices. As seen in figure 3, there is good aggreement between the two expressions. Excluding the smallest lattice, the $\kappa$ (certainly not $\Lambda$ ) seems to be

\footnotetext{
${ }^{2}$ See [2] for details.
} 
stable against a change in volume. For the largest volume $\kappa=0.5138_{-22}^{+16}$ and $0.5122_{-15}^{+15}$ for the cut and pole formulas, respectively. In this sense, the lattice data supports a vanishing zero momentum gluon propagator.
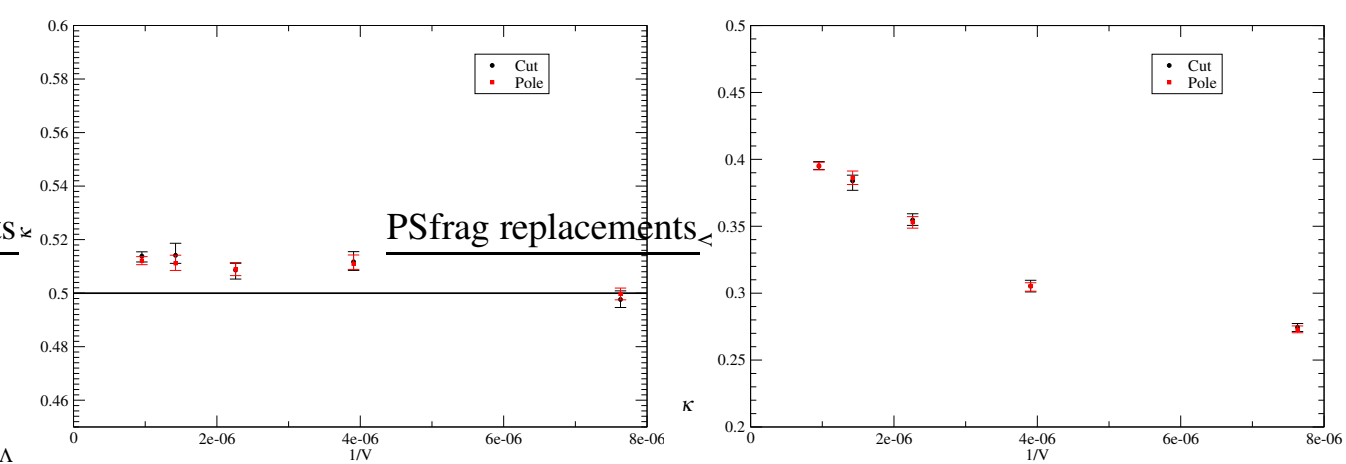

Figure 3: $Z_{c u t}$ and $Z_{\text {pole }}$ fitted parameters as function of lattice volume.

Let us now look at the behaviour of the propagator as a function of the volume. For the four lowest momenta values, the volume dependence can be seen in figure 4. To extrapolate the zero momentum gluon propagator we tried the formulas reported in table 2 . The results are a bit disapointing in the sense that all functional formulas produce good fits. From the table, one sees that the extrapolation is compatible with all types of zero momentum behaviour, from zero to infinite. Nevertheless, the results of table 2 will help to reduce the number of fitting formulas to be considered in the extrapolation of the full propagator.
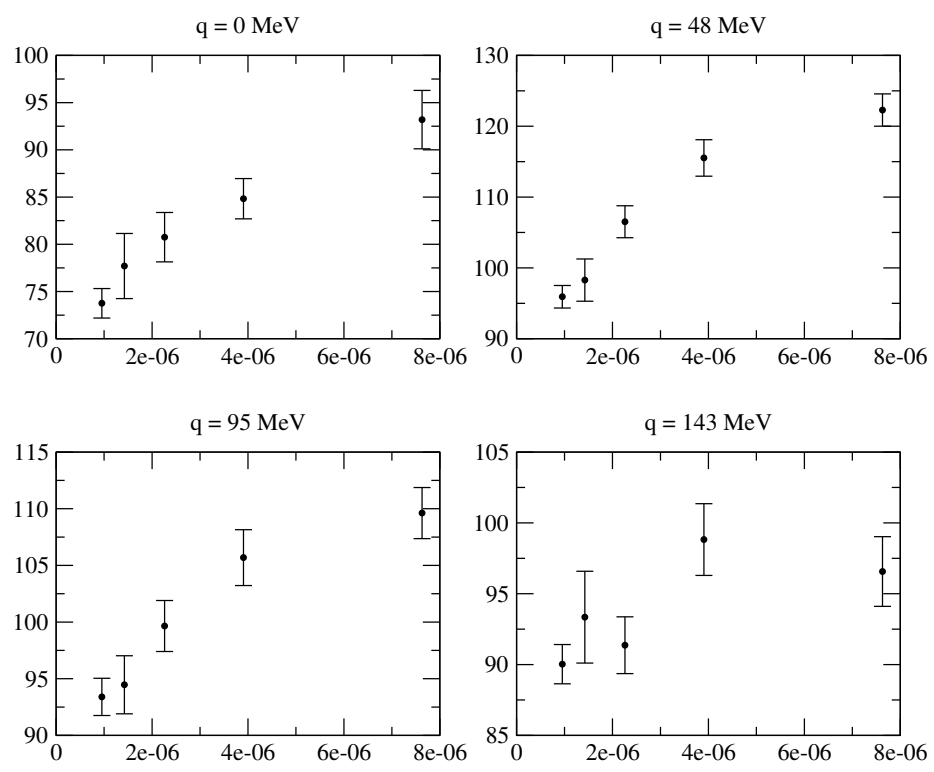

Figure 4: Propagator as a function of the inverse volume. 


\begin{tabular}{|lcl|}
\hline & $\chi^{2} /$ d.o.f. & Fitted Parameters \\
\hline$a+b x$ & 0.60 & $a=72.06, b=3.0 \times 10^{6}$ \\
$a+b x+c x^{2}$ & 0.24 & $a=69.52, b=5.1 \times 10^{6}, c=-2.5 \times 10^{11}$ \\
$a+b x^{c}$ & 0.10 & $a=53.96, b=1702, c=0.32$ \\
$a x^{b}$ & 0.12 & $a=325.4, b=0.11$ \\
$(a+b x) x^{c}$ & 0.13 & $a=2 \times 10^{-5}, b=418, c=-0.87$ \\
\hline
\end{tabular}

Table 2: Zero momenta propagator extrapolation, where $x=1 / \mathrm{V}$.

\section{Infinite volume extrapolation}

As a guide to the infinite volume extrapolation we take the results of the zero momentum gluon propagator. Since in the last fitting formula $a \sim 10^{-5}$, we will only consider the following fitting functions

$$
a+b x+c x^{2}, \quad a+b x^{c},
$$

and will fit only the timelike momenta. Moreover, each timelike momenta will be fitted separatly. From the two fitting formulas, the second one does not provide a smooth propagator. For certain momenta the fits do not converge or produce an infinite propagator. Therefore, from now on we will consider only the fits assuming a quadratic behaviour. Indeed, for the quadratic function, the extrapolated propagator looks rather smooth (see figure 5) and only less than $15 \%$ of the fits have a $\chi^{2} /$ d.o.f. $>2$.

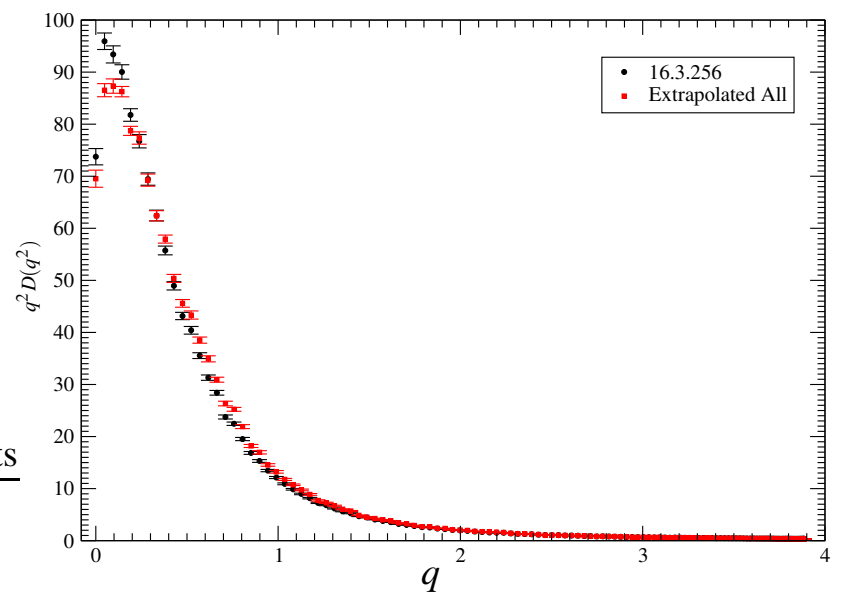

Figure 5: Extrapolated gluon propagator.

If one tries to repeat the study performed in $[1,2]$ it cames that the only fits which have acceptable $\chi^{2} /$ d.o.f. are the fits to the IR region. Probably, this is due to the inclusion of the data from the smallest lattice which looks different from all the other ones. The results of the IR fits are reported in table 3. Again, the lattice although pointing towards a vanishing zero momentum, it does not provide a definite answer. Looking at the $\kappa$ values, the fit to the analytical DSE solution is 
compatible with 0.5 . However, it is interesting that if one includes corrections to the above solution, the result agrees within errors with the figures estimated from the largest lattice, using the same procedure.

\begin{tabular}{|c|rcr|rrr|}
\hline & \multicolumn{3}{|c|}{ Extrapolated } & \multicolumn{3}{c|}{$16^{3} \times 256$} \\
& $\kappa$ & $\Lambda$ & $\chi^{2} /$ d.o.f. & $\kappa$ & $\Lambda$ & $\chi^{2} /$ d.o.f. \\
\hline$\left(q^{2}\right)^{2 \kappa}$ & $0.4993(7)$ & - & 0.37 & $0.4858(2)$ & - & 0.40 \\
\hline$\left(q^{2}\right)^{2 \kappa}\left(1+a q^{2}\right)$ & $0.5129(10)$ & - & 0.07 & $0.5070(50)$ & - & 0.44 \\
\hline$\left(\frac{q^{2}}{q^{2}+\Lambda^{2}}\right)^{2 \kappa}$ & $0.5198(2)$ & $438(1)$ & 1.29 & $0.5090(20)$ & $409(4)$ & 0.71 \\
\hline$\frac{\left(q^{2}\right)^{2 \kappa}}{\left(q^{2}\right)^{2 \kappa}+\left(q^{2}\right)^{2 \kappa}}$ & $0.5167(1)$ & $439(1)$ & 1.36 & $0.5077(17)$ & $409(4)$ & 0.69 \\
\hline
\end{tabular}

Table 3: IR fits. The errors on the extrapolated propagator are clearly underestimated.

\section{Conclusions}

We have performed a first study of the volume dependence of the gluon propagator. Although the lattice data seems to favour $\kappa \geq 0.5$, we are not able yet to provide a clear answer concerning the behaviour of the gluon propagator at zero momentum. In what concerns the IR region, our data shows a mild but measurable dependence with the volume. This is not in aggreement with a recent similar study of the DSE equations on a tours $[4,5]$.

\section{Acknowledgements}

We would like to thank A. C. Aguillar, C. S. Fischer, A. G. Williams, J. I. Skullerud, P. Bowman and D. Leinweber for fruitful and inspiring discussions.

\section{References}

[1] O. Oliveira, P. J. Silva, AIP Conf. Proc. 756 (2005) 290 [hep-lat/0410048 ].

[2] P. J. Silva, O. Oliveira, these proceedings [hep-lat/0509034 ].

[3] P. J. Silva, O. Oliveira, Nucl. Phys. B690(2004) 177 [hep-lat/0403026 ].

[4] C. S. Fischer, B. Gruter, R. Alkofer, hep-ph/0506053 .

[5] C. S. Fischer, these proccedings [hep-lat/0509031 ]. 